\title{
Diacronie
}

Studi di Storia Contemporanea

$N^{\circ} 27,3$ | 2016

Stato, costituzione e democrazia

\section{Maria Roberta Novielli, Animerama. Storia del cinema d'animazione giapponese}

\section{Sabrina Bassi}

\section{(2) OpenEdition}

Journals

Edizione digitale

URL: http://journals.openedition.org/diacronie/4220

DOI: $10.4000 /$ diacronie.4220

ISSN: 2038-0925

Editore

Association culturelle Diacronie

Notizia bibliografica digitale

Sabrina Bassi, « Maria Roberta Novielli, Animerama. Storia del cinema d'animazione giapponese», Diacronie [Online], № 27, 3 | 2016, documento 23, online dal 29 septembre 2016, consultato il 10 décembre 2020. URL : http://journals.openedition.org/diacronie/4220 ; DOI : https://doi.org/10.4000/ diacronie. 4220 


\section{Diacronie}

\section{RECENSIONE:}

\section{Maria Roberta NOVIELLI, Animerama. Storia del cinema d'animazione giapponese, Venezia, Marsilio, 2015, 287 pp.}

a cura di Sabrina BASSI *

Maria Roberta Novielli, docente di discipline legate al cinema e alla letteratura giapponese presso l'Università Ca'Foscari di Venezia, già autrice di monografie sul cinema del Giappone quali Storia del cinema giapponese ${ }^{1}$, Metamorfosi. Schegge di violenza nel nuovo cinema giapponese ${ }^{2}$ e Lo schermo scritto. Letteratura e cinema in Giappone 3 , offre al pubblico una nuova opera legata al cinema e al Giappone: questa volta si tratta del cinema di animazione che, escluso dal volume Storia del cinema giapponese per una scelta pratica e di chiarezza 4 , trova ora spazio in questo nuovo scritto.

Animerama. Storia del cinema d'animazione giapponese già dal titolo ci proietta nel mondo dell'Estremo Oriente:

Il titolo scelto per questo volume, è un omaggio alla pioneristica trilogia erotica ideata da TezukaOsamu e dal suo incredibile team di animatori tra il 1969 e il 1973 $[\ldots]^{5}$.

${ }^{1}$ NOVIELLI, Maria Roberta, Storia del cinema giapponese, Venezia, Marsilio, 2001.

2 ID., Metamorfosi. Schegge di violenza nel nuovo cinema giapponese, Castello di Serravalle, Epika, 2010.

3 ID., Lo schermo scritto. Letteratura e cinema in Giappone, Venezia, Cafoscarina, 2012.

4 Intervista a Marta Novielli a cura di Giuseppe Ferro,

URL: < http://www.asakusa.it/giappone_extra/interviste/maria_roberta_novielli.html > [consultato il 14 agosto 2015];

URL: < http://www.indie-eye.it/cinema/covercinema/animerama-storia-del-cinemadanimazione-giapponese-una-conversazione-con-maria-roberta-novielli.html > [consultato il 14 agosto 2015].

5 NOVIELLI, Maria Roberta, Animerama. Storia del cinema d'animazione giapponese, Venezia, Marsilio, 2015, p. 12 
Scopo di questo volume è quello di fornire un racconto coinvolgente ed esaustivo dello sviluppo di uno dei prodotti culturali più grandi e conosciuti attualmente in Occidentedel paese del Sol Levante.Il libro consta di diversi elementi: le trame, i personaggi, le storie... Ma, anche e soprattutto, mostra come le idee siano inscindibili dalla storia del Giappone e dalle sue tradizioni, poiché parlare di animazione significa parlare dell'arte, della cultura e della società giapponese.

Affrontare un tale discorso sulla terra nipponica ci porta ad analizzare un mondo che, ancora oggi, soggiace al fascino dell'esotismo in cui Maria Roberta Novelli, ci accompagna in un viaggio attraverso le epoche e l'anima di un Giappone in continua evoluzione.

L'autrice, prima di parlare direttamente e in maniera esaustiva di animazione, ci fa conoscere tutte quelle strutture volte al divertimento popolare che anticipano quello che sarà il cinema.

Così, come per l'occidente il cinemanasce anche dalla lanterna magica, dallo Zoopraxiscopio di Muybridge - e da tante meno note invenzioni che si sviluppano a cavallo tra la fine dell'Ottocento e l'inizio del Novecento - così l'animazione giapponese, trova le proprie radici in teatrini, lanterne e spettacoli di strada.

Gli Emakimono possono essere considerati i veri precursori dell'immagine animata:«consistevano in immagini e testi dipinti su seta o carta, utilizzati già nell’XI secolo» ${ }^{6}$ che venivano, oltre alla fruizione privata, srotolati e commentati da girovaghi (assimilabili ai nostri cantastorie) per narrare racconti al pubblico.Successivamente, il Kagee, il teatro delle ombre, ed una personalizzazione nipponica della lanterna magica (chiamata utsushie) importata dall'Olanda sul finire del Diciottesimo secolo, divengono i primi strumenti e le prime realtà di fruizione collettiva di spettacoli di animazione.

L'autrice si sofferma soprattutto sui momenti di confronto, di assimilazione tra la cultura giapponese e, conseguentemente, anche tra i suoi autori e quelli che sono i rappresentanti culturali del mondo occidentale.

Un esempio indicativo è l'ispirazione frequente che autori e animatori traggono dai racconti di Hans Christian Andersen, come Murata Yasuji con il suo La coda del re (1949), rivisitazione de I vestiti nuovi dell'imperatore in veste animale: in questo caso, si tratta del principe delle volpi nato senza coda. Altro esempio è la famosa favola de $L a$ piccola fiammiferaia che ispira due opere animate a silhouette del 1947: un omonimo corto di Yamamoto Sanae e Sogno di una notte nevosa di ŌfujiNoburō.

Le contaminazioni tra il paese nipponico e l'Occidente sono innumerevoli.

${ }^{6}$ NOVIELLI, Maria Roberta, Animerama, cit., p. 17. 
Risulta fondamentale, in questo senso, anche l'occupazione americana, che contribuisce alla nascita di opere animate dalle favole occidentali, ma non solo: le forze di occupazione USA promuovono film d'animazione che trattino lo sport, in particolare il baseball. Così nasce Il mio baseball (1948) per la regia di Asano Megumi, primo film d'animazione giapponese a colori.

Anche il personaggio leggendario di Momotarō è protagonista di diverse avventure, tra cui una serie di pellicole create e prodotte durante il periodo bellico come propaganda militare.

Emblematico risulta nel 1943, il film d'animazione Le aquile dei mari di Momotarō che fu creato sotto richiesta del Ministero della marina per dare rilievo, ancora una volta, al glorioso attacco a Pearl Harbor.

Tuttavia, molti nomi [...] sono poco noti in Occidente e perciò questo volume ha l'ambizione di suggerire nuove visioni e l'approccio ad animazioni anche molto distanti dalmainstream $[\ldots . .]^{7}$

come viene sottolineato in questa parte della premessa di Maria Roberta Novielli, lo scopo è anche quello di far conoscere nuove storie e nuove realtà ad un Occidente ancora troppo ignaro di ciò che il Giappone ha saputo sperimentare e conoscere anche prima dell'Europa.

Più volte ci vengono presentati, in diversi capitoli e in un intero paragrafo dedicato solo a loro, tutte quelle opere che vengono considerate indipendenti e di sperimentazione.

Qui scopriamo l'ennesimo filo rosso che collega il mondo giapponese a quello Occidentale o, più precisamente, a quello americano: Tanaami Keiichi (già artista, illustratore, fumettista ed esponente della pop art) incontra nel 1967 Andy Warhol e, tornato al lavoro sull'animazione, non può fare a meno di "portare con sé" il bagaglio culturale americano.

Un forte impulso alla creazione di storie attinenti al mondo alieno giunse dal mondo del manga già dall'immediato dopoguerra soprattutto dalle opere del suo più importante autore, poi creatore di serie animate: Tezuka Osamu, definito "il dio del manga" $[\ldots]^{8}$

7 NOVIELLI, Maria Roberta, Animerama, cit., p. 11

${ }^{8}$ NOVIELLI, Maria Roberta, Animerama, cit., p. 98. 
colui che introdurrà nel mondo dell'animazione il famosissimo Astro Boy (19521968), antesignano di quella stirpe di robot combattenti in cui rientrano opere molto diverse tra loro come Gundam il guerriero meccanico (regia di Tomino Yoshiyuki, 1978)9 ed il post-apocalittico Neon Genesis Evangelion (creato da Anno Hideaki, ${ }_{1995)^{10}}$, in grado di incarnare lo spirito del tempo, scosso dal terrore che si era impadronito della popolazione a seguito dell'attentato del 20 marzo 1995, in cui gli adepti di una setta depositarono sacchi contenenti gas nervino in diversi punti della metropolitana di Tokyo ${ }^{11}$.

Seguendo un percorso cronologico, affrontiamo tutte le fasi dello sviluppo dei film animati giapponesi passando anche attraverso il dissestato percorso dell'erotico: dai primi manga "a luci rosse" fino alla produzione degli hentai, o «produzione animata pornografica destinata al mercato video» ${ }^{12}$ sostenuta dall'home video degli anni Ottanta.

Un mondo popolato non solo da autori, registi ed animatori, ma anche da case di produzione e associazioni di autori; Novielli ci racconta così anche la nascita della Tōei, creata da Ōkawa Hiroshi, e dell'ancor più famoso Studio di Miyazaki Hayao e Takahata Isao.

L'autrice ci propone, di volta in volta, quelli che sono i capisaldi animati di un determinato periodo, momento storico o argomento.

Camminiamo, così, con delicatezza, fra le favole di Miyazaki Hayao come Porco Rosso $^{13}$, La principessa Mononoke ${ }^{14}$ o Il mio vicino Totoro ${ }^{15}$ per poi esplorare storie antiche e a noi sconosciute come Lo sfaticato va al castello Ryūgū (di Kimura Hakusan, 1925) ${ }^{16}$ o le innumerevoli avventure del personaggio leggendario Momotarō fino ad arrivare alle nuove generazioni di animazione in cui si sperimenta, come già in passato veniva fatto, e si creano nuove forme e nuovi mezzi di comunicazione di immagini in movimento.

9 YOSHIYUKI, Tomino, Kidō Senshi Gandamu, Nippon Sunrise, Giappone, 1979-1980, 43 episodi da 22'.

10 HIDEAKI, Anno, Shin seiki Evangerion, TV Tokyo - Nihon Ad Systems - Production I.G Studio Ghibli, Giappone, 1995-1996, 26 episodi da 23'.

${ }^{11}$ Sul tema si vedano le testimonianze raccolte nel libro di Murakami. MURAKAMI, Haruki, Underground. Racconto a più voci dell'attentato alla metropolitana di Tokyo, Torino, Einaudi, 2003.

${ }^{12}$ NOVIELLI, Maria Roberta, Animerama, cit., p. 258.

${ }^{13}$ MIYAZAKI, Hayao, Kurenai no buta, JAL - Nibariki - NTV, Giappone, 1992, 94'.

14 MIYAZAKI, Hayao, Mononoke-hime, DENTSU - Nibariki - NTV, Giappone, 1997, 134'.

${ }^{15}$ MIYAZAKI, Hayao, Tonari no Tororo, Studio Ghibli, Giappone, 1988, 86’.

${ }^{16}$ HAKUSAN, Kimura, Kanimanji engi, Asahi Kinema Gomei, Giappone, 1925, 11'. 
Dai progetti più famosi ed ambiziosi come Animatrix (del 2003 creato da un collettivo di autori, su diretta ispirazione del film The Matrix dei fratelli Wachowski) ${ }^{17}$ fino ad arrivare alle installazioni di Tabaimo ed ai suoi lavori dedicati al rapporto tra interni ed esterni; Dolefullhouse (2007) perfetta rappresentazione del messaggio dell'autrice, dove

[...] lo studio dello spazio si concentra sulle prospettive, mentre una mano posiziona piccoli mobili in miniatura in una casa per le bambole, indicando quali diverse percezioni lo spazio ci induca ad avere della realtà ${ }^{18}$.

Il linguaggio utilizzato da Maria Roberta Novielli è chiaro e diretto, coinvolge il lettore nel racconto e nel percorso fra le epoche del cinema giapponese.

Unico difetto risulta, per un "non addetto ai lavori" del cinema e dell'animazione giapponese, l'utilizzo - pur non frequente - di terminologia più specifica, che in ogni caso, non pregiudica la lettura e la comprensione da parte di un lettore esterno.

Fondamentale risulta l'inserimento di un glossario, che aiuta coloro che non conosco la lingua giapponese ad addentrarsi in questa terminologia nuova e ricorda al lettore i significati dei termini che l'autrice spiega nel corso dell'opera.

L'opera riesce, senza dubbio, nel suo scopo: creare una storia dell'animazione giapponese, dai suoi albori fino agli sviluppi più recenti, passando attraverso epoche storiche, autori e contesti culturali diversi.

Ciò che distingue il volume di Novielli, rispetto ad altri autori, è la capacità di contemplare nel proprio studio, non solo la produzione animata in lungometraggi, ma anche quella dei medio e cortometraggi.

Un'analisi precisa e puntuale dello sviluppo dell'animazione anche attraverso il vissuto e le motivazioni degli autori della produzione animata giapponese.

Il viaggio che ci propone l'autrice ci porta a scoprire nuovi autori, mangaka (termine con cui si definisce l'autore dei manga), animatori e a scoprire e riscoprire sotto una nuova luce le storie degli autori più famosi.

17 WACHOWSKI, Lana, WACHOWSKI, Andy, The Matrix, Warner Bros. Pictures, Stati UnitiAustralia, 1999, 136'.

${ }^{18}$ NOVIELLI, Maria Roberta, Animerama, cit., p. 247. 


\section{* L'autore}

Sabrina Bassi è studentessa del corso di Laurea in Discipline delle arti, della musica e dello spettacolo (DAMS) dell'Università di Bologna.

URL: < http://www.studistorici.com/progett/autori/\#Bassi >

\section{Per citare questo articolo:}

BASSI, Sabrina, «Recensione: Maria Roberta NOVIELLI, Animerama. Storia del cinema d'animazione giapponese, Venezia, Marsilio, 2015, 287 pp.», Diacronie. Studi di Storia Contemporanea : Stato, costituzione e democrazia, 29/09/2016,

URL:<http://www.studistorici.com/2016/09/29/bassi_numero_27/ >

\section{Diacronie Studi di Storia Contemporanea $\beta$ www.diacronie.it}

Risorsa digitale indipendente a carattere storiografico. Uscita trimestrale.

\section{redazione.diacronie@hotmail.it}

Comitato di redazione: Jacopo Bassi - Luca Bufarale - Elisa Grandi - Antonio César Moreno Cantano - Deborah Paci - Fausto Pietrancosta - Alessandro Salvador - Matteo Tomasoni - Luca Zuccolo autorizzata, nei limiti previsti dalla legge. 